\title{
Dépendance envers l'alcool Intérêt et limites des modèles expérimentaux
}

On sait maintenant reproduire sur l'animal deux aspects de la dépendance alcoolique: la dépendance physique (syndrome de sevrage) et la dépendance psychologique (recherche d'alcool). Certaines données suggèrent que la dépendance pourrait être liée aux modifications physico-chimiques des membranes: tout d'abord une fluidification par effet direct de l'alcool, puis, probablement par un phénomène adaptatif, une rigidification croissante associée à une modification des phospholipides. Les recherches thérapeutiques actuelles s'orientent sur des produits agissant sur les membranes ou sur le métabolisme des neurotransmetteurs sérotoninergiques.

\section{Bernard Le Bourhis \\ Docteur es sciences. \\ Chef du service de sécurité alimentaire-pharmacologie}

\section{RÉFÉRENCES}

1. Essig CF, Lam RC. Convulsions and hallucinating behaviour following alcohol withdrawal in the dog. Arch Neurol 1968; 18 : 626-32.

2. Victor M, Adams RD. The effects of alcohol on the nervous system. Res Publ Assoc Res Nerv Ment Dis 1953 ; 32 : 526-73.

3. Aufrère G. Contribution à l'étude de la dépendance à l'éthanol chez le rat: quantification du syndrome de sevrage et relation avec le protocole d'alcoolisation. Diplôme de l'École Pratique des Hautes Études (EPHE), Paris, 1982.

4. Le Bourhis B, Aufrère G. Pattern of alcohol administration and physical dependence. Alcohol Clin Exp Res 1983; 7 : 378-81.

5. Le Bourhis B, Aufrère G. Alcohol exposure pattern and physical dependence. Pharmacol Biochem Behav 1983 ; 18 : 511-4.

6. Le Bourhis B. Sur l'établissement de la dépendance des rats à l'égard de l'alcool. Physiol Behav 1977; 18 : 475-8.

7. Le Bourhis B, Aufrère G, Choquart D. Study of alcohol dependence in the rat. In : Begleiter H, ed. Biological Effects of Alcohol; New York : Plenum Publishing Corporation, 1980.

8. Le Bourhis B, Aufrère G. Precisions on the beverages free choice significance in behaviorally dependent rats. Santa Fe : 2nd Congress of the ISBRA, 1984.

\section{ADRESSE}

B. Le Bourhis : centre de recherche PernodRicard, 120, avenue du Maréchal-Foch, 94015 a dépendance vis-à-vis de l'alcool revêt deux aspects fondamentaux: physique et psychologique (ou comportemental), que l'on observe fréquemment chez l'homme. L'aspect physique correspond à l'ensemble des symptômes du syndrome de sevrage : tremblements, agitation, hallucinations, mouvements convulsifs, etc. L'aspect psychologique ou comportemental est caractérisé par la sensation de manque et la recherche de l'objet de dépendance, en l'espèce les boissons alcooliques; c'est le craving* des auteurs anglo-saxons.

La dépendance est-elle un phénomène typiquement humain? On l'a cru pendant longtemps.

En effet, seul l'homme est capable de commettre spontanément des erreurs alimentaires qui le conduisent à manger et à boire plus que de besoin. L'animal, au contraire, est doué d'une toxicophobie qui lui permet d'ajuster ses prises alimentaires à ses besoins et donc de stopper la consommation d'un aliment en deçà d'un seuil toxique.

L'étude expérimentale de la dépendance à l'égard de l'alcool, chez l'animal, nécessitait donc de créer des modèles l'obligeant à absorber des doses toxiques d'alcool. Une fois ces modèles mis en place, on a pu aisément induire la dépendance physique; la dépendance comportemen- tale fut mise en évidence plus tardivement.

Les modèles dont on dispose ont permis, d'une part, de faire progresser nos connaissances sur le mécanisme d'acquisition de la dépendance et, d'autre part, d'envisager quelques possibilités de traitement des alcooliques.

\section{L'intoxication alcoolique expérimentale}

Alcooliser des animaux ne pose pas de problèmes particuliers. Les intoxiquer, c'est-à-dire leur administrer une dose toxique d'alcool, autrement dit une dose supérieure à celle qu'ils peuvent dégrader, ce qui va donc nécessairement se traduire par une alcoolémie plus ou moins élevée, est beaucoup plus difficile.

Cette difficulté s'explique pour deux raisons : (a) la première est la toxicophobie dont nous avons déjà parlé plus haut; (b) la seconde est que les animaux d'expérience ont une capacité à oxyder l'alcool toujours supérieure à celle de l'homme (Tableau I, p. 347, possibilités de dégradation de l'éthanol dans différentes espèces animales). On ne peut pourtant pas imaginer rendre dépendants des ani* Craving : de l'anglais to crave, implorer avec
insistance. 


\begin{tabular}{|c|c|}
\hline \multicolumn{2}{|c|}{ Tableau I } \\
\hline \multicolumn{2}{|c|}{ POSSIBILITÉS DE DÉGRADATION DE L'ÉTHANOL } \\
DANS DIFFÉRENTES ESPËCES ANIMALES \\
\hline Espèces & Métabolisation \\
& (mg/kg/heure) \\
\hline & \\
Homme & $100-120$ \\
Chat & $130-150$ \\
Chien & $130-150$ \\
Lapin & $200-220$ \\
Cobaye & $200-220$ \\
Pigeon & $250-280$ \\
Rat & $300-400$ \\
Poulet & $300-400$ \\
Souris & $500-600$ \\
\hline
\end{tabular}

maux qui n'ont pas été «intoxiqués » par l'alcool.

Tenant compte de ces particularités, les expérimentateurs ont mis au point des méthodes d'alcoolisation dans lesquelles l'animal absorbe spontanément des quantités intoxicantes d'alcool (régime semi-liquide de Lieber) ou d'autres dans lesquelles la quantité absorbée est indépendante de la volonté de l'animal (gavage dans le cas des expériences aiguës ou de courte durée; perfusion, intragastrique, intraveineuse ou séjour en atmosphère alcoolisée dans le cas d'expérience de longue durée).

\section{Dépendance physique}

Cet aspect de la dépendance a été mis en évidence expérimentalement il y a plus de vingt ans, chez le singe. Mais la première description des réactions de sevrage est due à Essib et Lam expérimentant sur des chiens[1]. L'alcool était administré, au moyen d'une canule intragastrique, toutes les quatre heures pendant deux semaines. Lors du sevrage on observait un tableau clinique comparable à celui du syndrome d'abstinence chez l'homme: tremblements, spasmes, cris et convulsions, parfois mortelles, tel qu'il a été décrit par Victor $\mathrm{m} / \mathrm{s} n^{\circ} 6$ vol. 4, juin 88 facilitant ou déclenchant la crise (choc électrique, stimulation acoustique, agent pharmacologique).

On a aussi utilisé d'autres signes de la crise de sevrage tels que la perte de poids, ou l'hypothermie. Ces méthodes présentent toutes les mêmes inconvénients: ne s'appliquer qu'aux animaux présentant le signe étudié, ne tenir aucun compte des autres signes.

En fait une bonne évaluation de la sévérité du syndrome de sevrage devrait intégrer tous les signes susceptibles d'apparaître. En outre, il faut être conscient du fait que le passage d'un signe à un autre, estimé plus grave, ne repose que sur un critère clinique pas nécessairement quantifiable.

De nombreux chercheurs ont tenté cette démarche mais la diversité des protocoles utilisés montre bien leur inadéquation à couvrir l'ensemble du syndrome de sevrage.

D'une manière générale, la sévérité du syndrome de sevrage est liée au niveau d'alcoolisation. Elle dépend essentiellement de la dose administrée et de la durée de l'intoxication. Par ailleurs, pour un même produit (dose $\times$ temps), elle dépend du protocole expérimental.

Aufrère, en 1982 [3] a étudié sur le rat l'influence du mode d'alcoolisation sur l'intensité du syndrome de sevrage, en faisant varier la dose et le temps de manière à ce que le produit reste constant. En fait, comme il utilisait une méthode d'alcoolisation continue par voie pulmonaire, pour laquelle il est difficile de connaître avec exactitude la quantité d'alcool absorbée, il a considéré les surfaces sous les courbes d'alcoolémie. Les rats séjournaient dans une atmosphère dont la teneur en alcool aug. mentait progressivement de 15 à $24 \mathrm{mg} /$ litre pendant une période allant de 9 à 32 jours selon les rats. Il a constaté que le syndrome de sevrage était d'autant plus sévère que la teneur en alcool de l'atmosphère avait été élevée rapidement [3]. Ce résultat laisse à penser que la consommation de fortes doses, engendrant une alcoolémie très élevée pendant une période courte, provoque un syndrome de sevrage plus sévère que la même consommation totale sous forme de doses plus 
modérées étalées sur une période plus longue. En d'autres termes, il semble qu'au cours d'une alcoolisation chronique, et indépendamment de la quantité totale absorbée, la dépendance apparaît plus ou moins rapidement selon la progression des doses administrées, progression qui peut être nulle dans le cas de l'administration d'une dose constante $[4,5]$. Transposé à l'alcoolisation de l'homme, ce résultat pourrait expliquer les différences de réponses observées chez les alcooliques méditerranéens, consommateurs parfois excessifs mais réguliers, et les alcooliques nordiques, buveurs irrréguliers mais absorbant des quantités souvent supérieures à celles régulièrement consommées par les précédents.

\section{Dépendance compartementale}

L'aspect comportemental de la dépendance a été mis en évidence, pour la première fois dans notre laboratoire, sur des rats soumis à une intoxication prolongée par inhalation de vapeur d'alcool [6]. Si l'on donne, à un rat, le choix entre de l'eau et une solution alcoolique à $10 \%$, il refuse d'absorber plus de 5 à $10 \mathrm{ml}$ de cette dernière, ce qui correspond à une ingestion de $\mathrm{l}$ à $2 \mathrm{~g} / \mathrm{kg}$ de poids corporel d'éthanol, nettement inférieure aux possibilités d'oxydation de son organisme (environ $8 \mathrm{~g} / \mathrm{kg} /$ jour). En le soumettant à une intoxication forcée et continue, par voie pulmonaire, on peut lui faire absorber, contre sa volonté, jusqu'à 12 ou $14 \mathrm{~g} / \mathrm{kg} /$ jour d'éthanol (figure 1, p. 349). En dépit de cela, mis ultérieurement en situation de choix de boisson, il s'abreuve presque exclusivement d'eau, bien qu'il manifeste tous les symptômes de la dépendance physique. La toxicophobie de l'alcool, due au moins en partie à une palatabilité ${ }^{*}$ négative, se manifeste toujours et empêche l'animal de prendre le remède qui le soustrairait aux effets de sa dépendance.

Nous avons donc imaginé un protocole dans lequel le rat est incité (par

* Palatabilité : sensation ol factive et gustative au niveau du palais. un assoiffement préalable) à boire de l'alcool (faute d'avoir une autre boisson) pendant la crise de sevrage qui survient 8 à 12 heures après cessation de l'intoxication. Dès le lendemain, on lui donne à nouveau le choix entre de l'eau et une solution alcoolique à $10 \%$ et on constate alors qu'il s'abreuve, presque exclusivement, de la solution alcoolique, ingérant ainsi 8 à $12 \mathrm{~g} / \mathrm{kg}$ /jour d'éthanol [7].

Ce choix d'alcool dure plusieurs semaines et correspond bien à un comportement de dépendance. En effet, on constate qu'un tel animal s'auto-entretient une alcoolémie variant de 0,1 à $1,5 \mathrm{~g}$ /litre. Par ailleurs, si on change la teneur 'en alcool de la solution alcoolique offerte en choix, l'animal module ses consommations de manière à ce que sa prise d'alcool reste à un niveau sensiblement constant. Enfin, si après plusieurs jours d'une telle situation de choix, on le sèvre d'alcool, on déclenche une réaction de sevrage [8].

Ce modèle correspond-t-il à celui de rats «alcooliques»? Sans aucun doute, non. Ainsi, contrairement à ce qui se passe souvent chez l'homme, la consommation volontaire et spontanée d'alcool, bien loin d'augmenter au cours de la période de choix, diminue progressivement et après cinq à sept semaines l'animal a progressivement « oublié » sa préférence pour la solution alcoolique. L'alcool n'a donc pas manifesté les propriétés renforçantes qu'on lui connaît chez l'homme

\section{Mécanisme d'installation de la dépendance: théorie des opioïdes}

Depuis plus de 20 ans, on a remarqué que l'administration d'alcool modifiait le métabolisme des neurotransmetteurs, notamment des catécholamines (dopamine et noradrénaline)[9], indolamines (sérotonine) [10]. Par ailleurs, on a montré [11] que ces neurotransmetteurs pouvaient se condenser, in vitro, avec l'acétaldéhyde, premier métabolite de l'éthanol, pour donner des composés à structure opioïde, proche de celle de la morphine et de la codéine : les tétrahydroisoquinolines (TIQ), les tétrahydropapavérolines (THP), les tétrahydrocarbolines. Comme on connaissait déjà l'existence de récepteurs cérébraux de la morphine, on a émis l'hypothèse que l'éthanol agirait lui aussi, de façon indirecte, sur ces récepteurs. Ce fut le point de départ de nombreuses recherches. On a alors constaté que les TIQ, les THP et les carbolines étaient hallucinogènes, qu'elles induisaient une forte dépendance et qu'elles augmentaient la consommation spontanée d'alcool. Cette piste de recherche bénéficia d'un nouvel intérêt lorsque, quelques années plus tard, on découvrit l'existence de pentapeptides d'origine endogène à structures opioïdes : les enképhalines et les endorphines qui se fixent sur les récepteurs de la morphine et des opiacés. Cette découverte modifia les conceptions classiques de la perception de la douleur. Un stimulus douloureux ou un stress provoquerait une décharge d'enképhalines et d'endorphines pour atténuer la douleur. $\mathrm{Si}$ on bloque les récepteurs de ces neuropeptides par un antagoniste spécifique tel que la naloxone, on augmente la sensation douloureuse, mais on réduit du même coup la consommation spontanée d'alcool. Les opioïdes provenant de la condensation de l'acétaldéhyde et de certaines amines biogènes pourraient bien être à l'origine des effets de l'alcool et de sa dépendance. De grands espoirs apparaissaient. Malheureusement, on n'a jamais pu montrer l'existence de ces substances dans le cerveau des animaux alcoolisés.

On estime actuellement que les TIQ les THP et les carbolines ont une durée de vie trop courte pour jouer un rôle important dans le déterminisme de la dépendance. Finalement, cette théorie est tombée en désuétude au profit de la théorie membranaire.

\section{Théorie membranaire}

L'action moléculaire de l'éthanol sur les membranes cellulaires a été l'objet de très nombreuses recherches au cours des dernières décennies. Il reste encore quelques points obscurs mais nos connaissances constituent maintenant un ensemble cohérent. Quelques articles récents [12-14] donnent une vue synthétique de la question. 

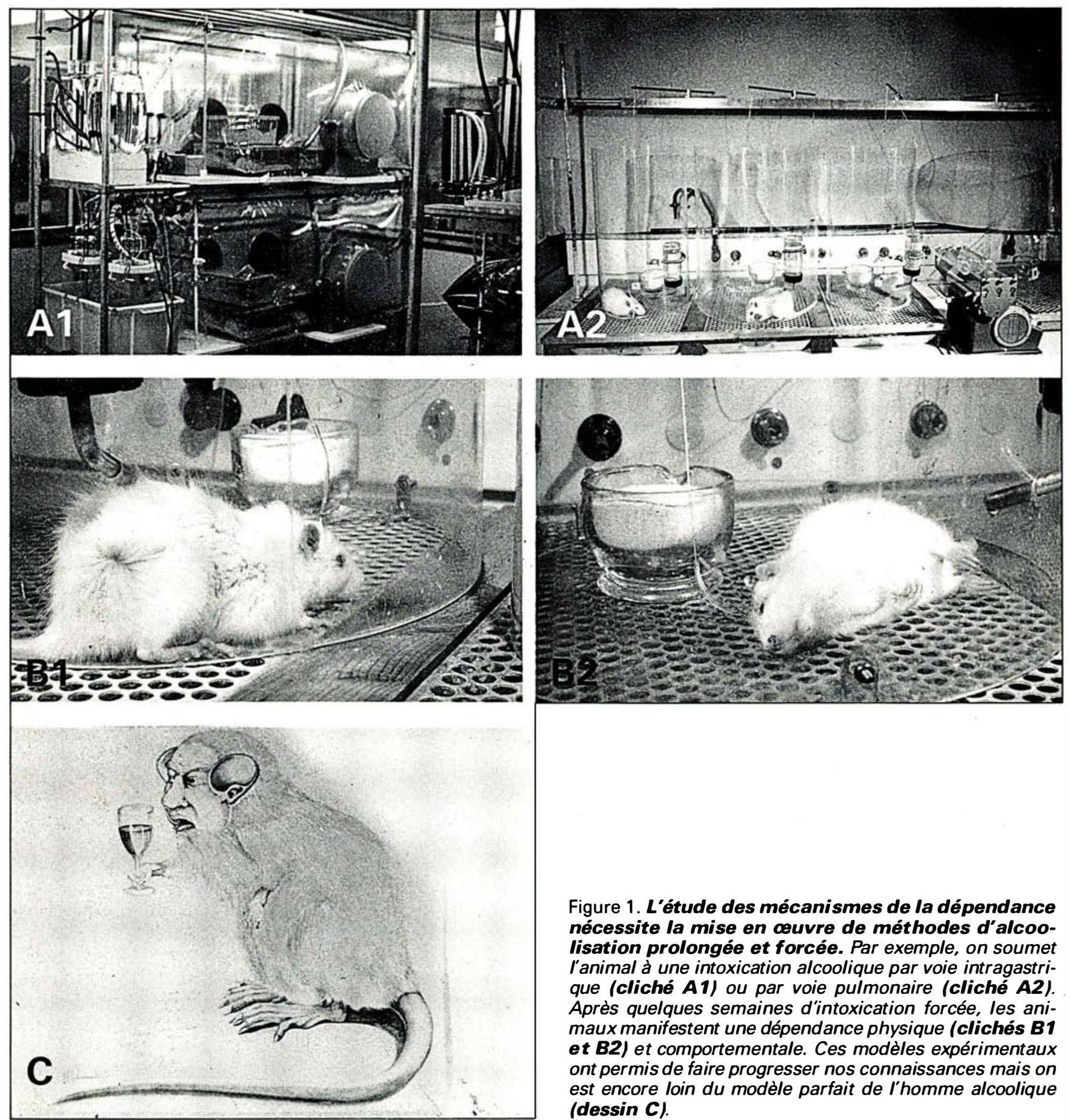

Figure 1. L'étude des mécanismes de la dépendance nécessite la mise en cuvre de méthodes d'alcoolisation prolongée et forcée. Par exemple, on soumet l'animal à une intoxication alcoolique par voie intragastrique (cliché A1) ou par voie pulmonaire (cliché A2). Après quelques semaines d'intoxication forcée, les animaux manifestent une dépendance physique (clichés B1 et B2) et comportementale. Ces modèles expérimentaux ont permis de faire progresser nos connaissances mais on est encore loin du modèle parfait de l'homme alcoolique (dessin C).

Toutes les membranes biologiques ont une structure globale commune : ce sont des assemblages de molécules de phospholipides et de protéines maintenues ensemble par des interactions non covalentes. Les molécules de lipides sont organisées en double couche et c'est cette $\mathrm{m} / \mathrm{s} n^{\circ} 6$ vol. 4, juin 88 bicouche qui constitue la structure de base de la membrane. La membrane se présente donc comme une mosaïque fluide [15] qui sert de barrière au flux de molécules hydrosolubles.

Les phospholipides membranaires sont constitués d'une tête polaire hydrophile et de deux queues hydrocarbonées hydrophobes. On conçoit évidemment que des variations de cette fluidité membranaire puisse fortement influer sur les propriétés de la membrane, en particulier modifier la nature et l'efficacité des récepteurs et d'une manière plus 


\section{RÉFÉRENCES}

9. Davis VE, Brown H, Huff JA, Cashaw JL. The alteration of serotonin metabolism to 5 Hydroxy tryptophol by ethanol ingestion in man. J Lab Clin Med 1967 ; 69 : 132-40.

10. Davis VE, Cashaw JL, Brown NA, Nicholas NL. Alteration of endogenous catecholamine metabolism by ethanol ingestion. Proc Soc Exp Biol Med 1967 ; 125 : 1140-3.

11. Davis VE, Walsh MJ. Alcohol, amines and alkaloïds: a possible biochemical basis for alcohol addiction. Science $1970 ; 167$. 1005-7.

12. Sun GY, Sun AY. Ethanol and membrane lipids. Alcohol Clin Exp Res 1985; 9: 164-80

13. Goldstein DB. Effect of alcohol on cellular membranes. Ann Emerg Med 1986; 15 1013-8.

14. Leonard E. Is ethanol a neurotoxin? The effects of ethanol on neuronal structure and function. Alcoholism 1986; 21 : 325-38.

15. Singer SJ, Nicolson GL. The fluid mosaic model of the structure of cell membranes. Science 1972 ; 175 : 720-31.

16. Chin JH, Parsons LM, Goldstein DB. Increased cholesterol content of erythrocyte and brain membranes in ethanol tolerant mice. Biochim Biophys Acta 1978; 513 : 358-63.

17. Littleton JM, John G. Synaptosomal membrane lipids of mice during continuous exposure to ethanol. J Pharmacol $1977 ; 29$ : 579-80.

18. Chin JH, Goldstein DB. Drug tolerance in biomembranes: a spin label study of the effects of ethanol. Science $1977 ; 196$ : 684-5.

19. Littleton JM, Jones G. Membrane lipids and ethanol tolerance in the mouse. The influence of dietary fatty acids composition. Life Sci 1980 ; 27 : 545-55.

20. Le Bourhis B, Beauge F, Aufrère G, Nordmann R. Membrane fluidity and alcohol dependence. Alcohol Clin Exp Res 1987; 10 : 337-42.

21. Beauge F. Communication personnelle 1987.

22. Rockman GE, Amit Z, Brown ZW, Bourque $\mathrm{C}$, Ogren SO. An investigation of the mechanisms of action of 5 hydroxytryptamine in the suppression of ethanol intake Neuropharmacology $1982 ; 21$ : 341-7.

23. Le Bourhis $B$, Uzan A, Aufrère G, Le Fur G. Effets de l'indalpine, inhibiteur spécifique de la recapture de la sérotonine sur la dépendance comportementale à l'éthanol et la prise volontaire d'alcool chez le rat. Ann Pharm Fr 1981 ; 39 : 11-20.

24. Le Bourhis B, Aufrère G, Eriksson CJP. Rôle de la sérotonine cérébrale dans la consommation volontaire d'alcool chez le rat. Les cahiers de l'IREB 1981 ; 5 : 19-23.

25. Boismare F, Daoust M, Moore N, et al. A homotaurine derivative reduces the voluntary intake of ethanol by rats : are cerebral GABA receptors involved? Pharmacol Biochem Behav 1984; 21 : 787-9.

26. Hillemand B, Lhuintre JP, Boismare F Daoust M, Moore M. Premier essai favorable du Nbis-homotaiurinate de calcium (AOTA Ca) dans le traitement de l'alcool-dépen- générale modifier la transmission de l'information.

La fluidité membranaire peut être mesurée par résonance magnétique nucléaire $(\mathrm{RMN})$, résonance paramagnétique d'électrons (RPE) ou par polarisation de fluorescence (PR).

D'une manière générale, les anesthésiques, et l'éthanol en particulier, agissent sur la membrane cellulaire en augmentant sa fluidité. Cet effet est facile à mettre en évidence in vitro et on a montré que le désordre membranaire était lié à la concentration d'alcool dans le milieu. On peut l'observer avec la plupart des membranes telles que celles des neurones, des hépatocytes ou des érythrocytes. Beaucoup d'anesthésiques manifestent un tel effet et leur action pharmacologique in vivo correspond bien à leur capacité à désorganiser la membrane et à leur pouvoir solvant des lipides. Bien qu'on ne puisse pas conclure avec certitude que cette action soit la cause de l'activité anesthésique, on peut émettre l'hypothèse que l'alcool agit d'abord sur les lipides membranaires. Évidemment cette activité fluidifiante est faible et très difficile à mettre en évidence, au moins après l'administration d'une dose aiguë.

Dans le cas d'une administration chronique, on a pu observer, chez l'animal, de profondes modifications de la structure membranaire. L'administration répétée d'alcool déclenche la mise en œuvre de mécanismes adaptatifs qui tendent à induire un effet opposé à celui provoqué par une dose unique d'alcool. Divers protocoles expérimentaux ont en effet démontré que, dans ces conditions, la membrane se rigidifiait progressivement.

Cette évolution de la viscosité est évidemment due à des modifications dans la composition chimique de la membrane. Dès 1978, on a montré [16] que la teneur membranaire en cholestérol d'érythrocytes de souris soumises à une absorption chronique d'alcool était augmentée. Cette observation est cohérente avec les modifications physiques de la membrane et on connaît le rôle que joue le cholestérol dans le contrôle de la fluidité membranaire. On sait aussi, depuis 1977, que l'alcoolisation chronique s'accompagne d'une modification de la nature des acides gras membranaires. On observe en particulier une baisse de la teneur en acides gras polyinsaturés et une augmentation de celle des acides gras saturés [17]. Ces modifications n'ont pas encore reçu d'explications pleinement satisfaisantes.

Comment l'alcool provoque-t-il la rigidité des membranes et l'accumulation de cholestérol et d'acides gras saturés? Certaines expériences in vitro suggèrent qu'il s'agit davantage d'un effet physique que d'un effet chimique, les acides saturés étant par nature plus «rigides» que les polyinsaturés; cette hypothèse n'est cependant pas encore prouvée.

Quoi qu'il en soit, il semble bien que ces modifications physico-chimiques des membranes cellulaires soient liées à l'apparition de la dépendance. Ainsi Chin et Goldstein ont montré que les membranes neuronales de certaines souches de souris «tolérantes » à l'alcool, étaient particulièrement résistantes à son effet fluidifiant [18]. De même si on donne à des souris un régime riche en acides gras saturés, elles deviennent peu à peu plus tolérantes à l'alcool [19]. Plus récemment, nous avons montré qu'il y avait une corrélation positive, hautement significative, entre la rigidité membranaire neuronale et la dépendance comportementale chez le rat [20].

Enfin il a été montré que les membranes érythrocytaires des alcooliques étaient plus rigides que celles des sujets normaux [21].

Bien que l'on ne dispose pas de preuves définitives de la relation causale entre les modifications des propriétés physico-chimiques des membranes et les effets biologiques de l'alcool, on peut estimer que cette causalité est néanmoins hautement probable. Cette théorie très séduisante permet de prendre en compte tous les effets psycho-physiologiques de l'éthanol : tolérance, dépendance physique et dépendance comportementale.

\section{Perspectives de traitement de la dépendance}

La liaison entre la composition lipidique membranaire et la dépendance constitue une voie de recherche inté- 
ressante. Peut-on prévenir la dépendance ou la guérir en administrant certains acides gras bien choisis, voire en modifiant quelque peu le régime alimentaire? Des recherches sont en cours dans ce domaine. En dépit des résultats positifs obtenus chez l'animal recevant un régime alimentaire strict, il n'est pas certain que ces recherches débouchent chez l'homme sur un résultat pratique intéressant. En effet, la composition chimique des membranes cellulaires est relativement stable et l'organisme s'efforce de conserver cette constance. En outre, on peut craindre qu'une modification des propriétés physicochimiques des membranes ne se solde par des déséquilibres outrepassant l'effet recherché. En revanche, on peut imaginer de trouver des agents pharmacologiques intervenant dans le métabolisme, la fixation ou le transfert des acides gras. Certains travaux réalisés dans ce sens donnent quelques espoirs de réussite. Une autre possibilité de traitement de la dépendance consiste à intervenir au niveau du métabolisme de certains neurotransmetteurs du système nerveux central.

On a en effet constaté, chez l'animal, que l'intoxication alcoolique chronique s'accompagnait de profondes modifications du renouvellement ou des concentrations cérébrales des principaux neurotransmetteurs, et tout particulièrement de ceux de la sérotonine. Selon les protocoles expérimentaux, les résultats varient, mais on peut dire que, dans l'ensemble, une intoxication alcoolique correspond à une baisse du taux de 5 hydroxytryptamine $(5 \mathrm{HT})^{*}$ cérébrale.

On peut donc imaginer que les traitements pharmacologiques tendant à augmenter la 5 hydroxytryptamine disponible auront un effet antidépendance. De fait, on a montré, sur divers modèles animaux, que les inhibiteurs de recapture de la sérotonine diminuaient la consommation volontaire d'alcool [22] et, même, manifestaient une action anti-dépendance curative ou préventive [23].

L'administration de précurseur de la

\footnotetext{
* 5 hydroxytryptamine $=5 H T=$ sérotonine.
} $m / s n^{\circ} 6$ vol. 4, juin 88
5HT produit le même effet [24]. En clinique humaine, deux inhibiteurs de recapture de 5HT (indalpine, zimélidine) ont manifesté une activité intéressante. Malheureusement ils n'ont trouvé aucune application, à cause de leurs effets secondaires néfastes. Actuellement un troisième composé du même type (fluoxétine) est en cours d'expérimentation aux États-Unis.

En France, Boismare et al. ont montré que certains dérivés de l'homotaurine réduisaient la prise volontaire d'alcool chez le rat [25]. Leur mécanisme d'action n'est pas définitivement établi ; il pourrait faire intervenir des modifications dans le fonctionnement du système gabaergique. L'un d'eux, le N-bis acétylhomotaurinate de calcium semble donner des résultats favorables chez l'homme [26].

\section{Conclusion}

Avant 1950, les recherches biomédicales sur l'alcool étaient à l'état de somnolence. La création de modèles animaux reproduisant les conditions d'intoxication chronique que l'on rencontre chez l'homme a permis de faire progresser nos connaissances à pas de géant.

Il n'existe pas de modèles d'«animaux alcooliques» au sens que l'on donne à ce terme en clinique humaine et il n'en existera peutêtre jamais. On sait malgré tout reproduire certains aspects de la dépendance à l'alcool et ces modèles sont d'un grand secours pour comprendre les mécanismes de cette dépendance, ceux de son acquisition et les possibilités de rompre cette dépendance.

La création d'un modèle expérimental de maladie humaine représente toujours pour le chercheur expérimentateur une satisfaction d'autant plus grande qu'il se rapproche au plus près de ce qui existe chez l'homme. En ce qui concerne un désordre comme la dépendance alcoolique chez l'homme, qui comporte des composantes physiologiques, génétiques, psychologiques et environnementales, il est à craindre malheureusement que l'on ne puisse jamais reproduire un modèle animal parfait de la situation humaine

\section{Summary}

On most species of laboratory animals, both aspects of dependence have been duplicated: physical dependence (withdrawal syndrom) and psychological dependence (craving for alcohol). The degree of dependence is difficult to assess; it has been shown, however, that it is to some extent correlated with the drinking habits. The mechanism of dependence acquisition has not been fully elucidated. The opioid theory has stirred up great expectations. Membrane phenomena are now favored. Ethanol fluidizes the bi-layer, leading to a series of physico-chemical changes which tend in turn to increase its rigidity. The withdrawal syndrome is the expression of this rigidity, which may then lead to an increasing intake. The search for antiabuse drugs is based on the study of membrane modifications and of the metabolism of certain neuro-transmitters (5HT, GABA).

\section{TIRÉS A PART}

B. Le Bourhis : centre de recherche PernodRicard, 120, avenue du Maréchal-Foch, 94015 Créteil, France. 